\title{
APPLICATION OF THE REALIZATION OF HOMOGENEOUS SOBOLEV SPACES TO NAVIER-STOKES*
}

\author{
LORENZO BRANDOLESE ${ }^{\dagger}$
}

\begin{abstract}
Molecule spaces have been introduced by Furioli and Terraneo [Funkcial. Ekvac., 45 (2002), pp. 141-160] to study some local behavior of solutions to the Navier-Stokes equations. In this paper we give a new characterization of these spaces and simplify Furioli and Terraneo's result. Our analysis also provides a persistence result for Navier-Stokes in a subspace of $L^{2}\left(\mathbb{R}^{3},\left(1+|x|^{2}\right)^{\alpha} d x\right)$, $\alpha<5 / 2$, which fills a gap between previously known results in the weighted- $L^{2}$ setting and those on the pointwise decay of the velocity field at infinity. Our main tool is the realization of homogeneous Sobolev spaces introduced by Bourdaud.
\end{abstract}

Key words. molecules, Hardy space, pointwise multipliers

AMS subject classifications. 36B40, 76D05, 35Q30

DOI. $10.1137 /$ S0036141004444408

1. Introduction. Consider the Navier-Stokes equations for a viscous incompressible fluid in the three-dimensional space and not submitted to external forces:

$$
\left\{\begin{array}{l}
\partial_{t} u+(u \cdot \nabla) u=\Delta u-\nabla p, \\
\nabla \cdot u=0 \\
u(x, 0)=a(x) .
\end{array}\right.
$$

Here $u=\left(u_{1}, u_{2}, u_{3}\right)$ is the velocity field and $p$ is the pressure, both defined in $\mathbb{R}^{3} \times$ $\left[0, \infty\left[\right.\right.$. Moreover, $\nabla \cdot u=\sum_{j=1}^{3} \partial_{j} u_{j}$ and $(u \cdot \nabla) u=\sum_{j=1}^{3} u_{j} \partial_{j} u$.

If $a \in L^{2}\left(\mathbb{R}^{3}\right)$, then we have known for a very long time that a weak solution to (NS) exists such that $u \in L^{\infty}(] 0, \infty\left[, L^{2}\left(\mathbb{R}^{3}\right)\right)$ and $\nabla u \in L^{2}(] 0, \infty\left[, L^{2}\left(\mathbb{R}^{3}\right)\right)$. If we know, in addition, that the initial datum is well localized in $\mathbb{R}^{3}$, then these conditions, of course, do not give us so much information on the spatial localization of $u(t)$ during the evolution. Then the natural problem arises of finding the functional spaces that would provide the good setting for obtaining such information. Several papers have been written on this topic; see, e.g., [14], [9], [10], [13], [1], [16] and the references therein. In particular, it was shown in [14] that the condition $a \in L^{2}\left(\mathbb{R}^{3},\left(1+|x|^{2}\right)^{\delta} d x\right)\left(0 \leq \delta \leq \frac{3}{2}\right)$ is conserved during the evolution, for a suitable class of weak solutions. Here and below, this weighted- $L^{2}$ space is equipped with the natural norm $\left(\int|a(x)|^{2}\left(1+|x|^{2}\right)^{\delta} d x\right)^{1 / 2}$. As far as we deal with data belonging to general weighted- $L^{2}$ spaces, it seems difficult to improve the upper bound on $\delta$.

When dealing with strong solutions to (NS) one can obtain sharper conclusions on the localization of $u$. For example, assuming that $a \in L^{1} \cap L^{2}\left(\mathbb{R}^{3}\right)$, He [13] proves, among other things, that $u(t)$ belongs to $L^{2}\left(\mathbb{R}^{3},\left(1+|x|^{2}\right)^{2} d x\right)$ at least in some time interval $[0, T], T>0$ (and uniformly in $[0,+\infty[$, under a supplementary smallness assumption). In a slightly different context, we would also like to mention the work of Miyakawa [17], in which it is shown that $u(x, t) \sim|x|^{-\alpha} t^{-\beta / 2}$ as $|x| \rightarrow \infty$ or $t \rightarrow \infty$,

\footnotetext{
*Received by the editors June 15, 2004; accepted for publication (in revised form) April 6, 2005; published electronically November 9, 2005.

http://www.siam.org/journals/sima/37-2/44440.html

${ }^{\dagger}$ Institut Camille Jordan Université Claude Bernard Lyon 1, 21 avenue Claude Bernard 69622 Villeurbanne Cedex, France (brandolese@math.univ-lyon1.fr).
} 
for all $\alpha, \beta \geq 0$ and $1 \leq \alpha+\beta \leq 4$, under suitable assumptions on $a$. The main tool here is the application of the contraction mapping theorem to the integral equation

$$
u(t)=e^{t \Delta} a-\int_{0}^{t} \nabla \cdot e^{(t-s) \Delta} \mathbb{P}(u \otimes u)(s) d s,
$$

where $e^{t \Delta}$ is the heat semigroup and $\mathbb{P}$ is the Leray-Hopf projector onto the solenoidal vector fields, defined by $\mathbb{P} f=f-\nabla \Delta^{-1}(\nabla \cdot f)$, where $f=\left(f_{1}, f_{2}, f_{3}\right)$. Note that (IE), together with the divergence-free condition $\nabla \cdot a=0$, is equivalent to (NS) under very general assumptions (see [11]).

If we compare the results on the spatial localization contained in [13] and [17], we see that Miyakawa's results seem to give a slightly better conclusion. Indeed, [17] tells us that the condition $a(x) \sim|x|^{-4}$ at infinity is conserved during the evolution (furthermore, $|x|^{-4}$ is known to be the optimal decay in the generic case), whereas, according to [13], the condition $u(t) \in L^{2}\left(\mathbb{R}^{3},\left(1+|x|^{2}\right)^{2} d x\right)$ only tells us, formally, that $u(t) \sim|x|^{-7 / 2}$ at infinity. Then there is a small gap between the results on the pointwise decay and those in the weighted- $L^{2}$ setting.

The first purpose of this paper is to obtain a persistence result in suitable subspaces of $L^{2}\left(\mathbb{R}^{3},\left(1+|x|^{2}\right)^{\alpha} d x\right)$, for all $0 \leq \alpha<\frac{5}{2}$, which, at least formally, will allow us to recover the optimal decay of the velocity field. To do this, rather than establishing a new theorem we shall give a new interpretation of a known result by Furioli and Terraneo on the molecules of the Hardy space [12]. More precisely, let us introduce the space $Z_{\delta}$ of functions (or vector fields) $f$ such that

$$
\begin{aligned}
f & \in L^{2}\left(\mathbb{R}^{3},\left(1+|x|^{2}\right)^{\delta-2} d x\right), \\
\nabla f & \in L^{2}\left(\mathbb{R}^{3},\left(1+|x|^{2}\right)^{\delta-1} d x\right), \\
\Delta f & \in L^{2}\left(\mathbb{R}^{3},\left(1+|x|^{2}\right)^{\delta} d x\right) .
\end{aligned}
$$

We provide such space with its natural norm. We will prove the following theorem (announced, in a weaker form, in [5]).

THEOREM 1.1. Let $\frac{1}{2} \leq \delta<\frac{9}{2}$ and let $a \in Z_{\delta}$ be a solenoidal vector field. Then there exists $T>0$ such that (IE) possesses a unique strong solution $u \in C\left([0, T], Z_{\delta}\right)$.

The restriction $\delta<\frac{9}{2}$ is consistent with the spatial spreading effect of the velocity field described, e.g., in [6]: we cannot have $u \in C\left([0, T], Z_{9 / 2}\right)$ unless the initial data have some symmetry properties. As we shall see, the elements of $Z_{\delta}$ are $o\left(|x|^{-\delta+1 / 2}\right)$ at infinity. Hence the correspondence between this result and those on the pointwise decay is not merely formal. The condition $\delta \geq \frac{1}{2}$ is physically reasonable since it prevents $u \rightarrow \infty$ as $|x| \rightarrow \infty$.

In section 3 we show that Theorem 1.1 is essentially equivalent to (but slightly improves) the result by Furioli and Terraneo [12]. Their motivation was different and this is probably the reason why the relation between their space of molecules $X_{\delta}$ (defined below) and the more natural space $Z_{\delta}$ is not found in [12]; motivated by the problem of the unicity of mild solutions in critical spaces (i.e., homogeneous spaces of degree -1), they studied the Navier-Stokes equations in $\Delta^{-1} \mathcal{H}^{1}$, which is the space made of all distributions vanishing at infinity and such that their Laplacian belongs to the Hardy space $\mathcal{H}^{1}\left(\mathbb{R}^{3}\right)$. As discussed also in [15], such space gives a useful insight of solutions to the Navier-Stokes equations. Indeed, if a solution $u$ satisfies $\Delta u \in \mathcal{H}^{1}\left(\mathbb{R}^{3}\right)$, then $u \in L^{3}\left(\mathbb{R}^{3}\right), \nabla \otimes u \in L^{3 / 2}$, and $(u \cdot \nabla) u \in \mathcal{H}^{1}\left(\mathbb{R}^{3}\right)$ (this is a consequence of the so-called div-curl lemma as stated in [7]). Furthermore, $\nabla p \in \mathcal{H}^{1}\left(\mathbb{R}^{3}\right)$ (this follows from the classical relation $\Delta p=\sum_{h, k=1}^{3} \partial_{h} \partial_{k}\left(u_{h} u_{k}\right)$ and 
the boundedness of the Riesz transforms in the Hardy space). Thus, the three terms which contribute to $\partial_{t} u$ in (NS) have the same regularity.

Moreover, the Hardy space has a very simple structure, due to its well-known atomic decomposition. Hence, solving the equations in $\Delta^{-1} \mathcal{H}^{1}$ yields a natural decomposition of the flow into simple "building blocks." Furioli and Terraneo considered the converse problem of studying the evolution of each building block. The result of [12] essentially states that if $\Delta u$ is a molecule of the Hardy space (in a sense close to that of Coifman and Weiss [8]) at the beginning of the evolution, then this property remains true for a certain time. To do this they introduced, for $\delta>\frac{3}{2}$, the space $X_{\delta}$ defined as the set of all tempered distributions $f$ vanishing at infinity such that $\Delta f \in L^{2}\left(\mathbb{R}^{3},\left(1+|x|^{2}\right)^{\delta} d x\right)$ and $\int x^{\alpha} \Delta f(x) d x=0$ for all $\alpha \in \mathbb{N}^{3}$ such that $\alpha_{1}+\alpha_{2}+\alpha_{3}<\delta-\frac{3}{2}$. The norm of $X_{\delta}$ is defined by

$$
\|f\|_{X_{\delta}}^{2} \equiv \int|\Delta f(x)|^{2}\left(1+|x|^{2}\right)^{\delta} d x
$$

Furioli and Terraneo's theorem then is stated as Theorem 1.1, with $X_{\delta}$ instead of $Z_{\delta}$, and with the additional restrictions $\frac{3}{2}<\delta<\frac{9}{2}$ and $\delta \neq \frac{5}{2}, \frac{7}{2}$. The condition $\delta>\frac{3}{2}$ is important for the embedding $X_{\delta} \subset \Delta^{-1} \mathcal{H}^{1}$. However, P.-G. Lemarié-Rieusset noticed that the condition $\delta \neq \frac{5}{2}, \frac{7}{2}$ can be removed. Their paper is technical and relies on the theory of local Muckenhoupt weights.

The second purpose of this paper is to provide a simpler proof of their result. Indeed, in section 4 we remark that the Fourier transform of $X_{\delta}$ is closely related to the realization "à la Bourdaud" [3] of the homogeneous Sobolev space $\dot{H}^{\delta}$. The operators involved in (IE) turn out to be Fourier pointwise multipliers of the realized spaces. Therefore the estimates that are needed to establish the boundedness of the bilinear operator $B(u, v)(t)=\int_{0}^{t} \nabla \cdot e^{(t-s) \Delta} \mathbb{P}(u \otimes v)(s) d s$ in $C\left([0, T], X_{\delta}\right)$ become very natural. The conclusion of the proof is a simple application of the contraction mapping theorem.

The idea of using Bourdaud's results on realized spaces in this way (or analogous results by Youssfi [20] for the realized homogeneous Besov spaces) seems to be new. Since this argument does not directly rely on the divergence-free condition or the matricial structure of $\mathbb{P}$, it can be easily applied to more general equations. Moreover, we feel that providing evidence of the relation between the localization problem of the velocity field and Furioli and Terraneo's molecules provides a better understanding of [12].

The spatial localization of the velocity field in different weighted-Lebesgue spaces is studied in [19]. After the first version of this paper was completed, the author was notified by H.-O. Bae and B. J. Jin that their preprint [2] also improves the spatial decay results of [13] and [14] and provides solutions to (NS) in $L^{2}\left(\mathbb{R}^{3},\left(1+|x|^{2}\right)^{\alpha} d x\right)$, $0 \leq \alpha<\frac{5}{2}$. Their method is a refinement of the weighted estimates of $\mathrm{He}$ and Xin [14] and is quite different than ours. The assumptions on the data are also different: Bae and Jin deal with less regular data, but they put more stringent assumptions on their spatial localization.

2. Some properties of the space $Z_{\delta}$. For $\delta \geq 0$ we introduce the space $L_{\delta}^{2}$ of all functions $f \in L^{2}\left(\mathbb{R}^{3},\left(1+|x|^{2}\right)^{\delta} d x\right)$ such that $\int x^{\alpha} f(x) d x=0$ for all $\alpha \in \mathbb{N}^{3}$, with $0 \leq|\alpha|<\delta-\frac{3}{2}$ (where $|\alpha|=\alpha_{1}+\alpha_{2}+\alpha_{3}$ ). There are of course no moment conditions for $0 \leq \delta \leq \frac{3}{2}$. Note that $L_{\delta}^{2}$ is well defined because of the embedding of $L^{2}\left(\mathbb{R}^{3},\left(1+|x|^{2}\right)^{\delta} d x\right)$ into $L^{1}\left(\mathbb{R}^{3}, w_{\delta}(x) d x\right)$, for $\delta>\frac{3}{2}$. Here and below, for $\delta>\frac{3}{2}$, 
we set $w_{\delta}(x)=(1+|x|)^{[\delta-3 / 2]}$ if $\delta-\frac{3}{2} \notin \mathbb{N}$ (where [.] denotes the integer part), and $w_{\delta}(x)=(1+|x|)^{\delta-5 / 2}$ otherwise.

Lemma 2.1. Let $\delta \geq 0$. We have $f \in L_{\delta}^{2}$ if and only if $f$ can be decomposed as

$$
f=g+\sum_{j=0}^{\infty} f_{j},
$$

where $g$ and $f_{j}$ belong to $L^{2}\left(\mathbb{R}^{3}\right), \operatorname{supp} g \subset\{|x| \leq 1\}$, $\operatorname{supp} f_{j} \subset\left\{2^{j-1} \leq|x| \leq 2^{j+1}\right\}$, and

$$
\begin{aligned}
& \left\|f_{j}\right\|_{2} \leq \epsilon_{j} 2^{-j \delta}, \quad \text { with } \quad \epsilon_{j} \in \ell^{2}(\mathbb{N}), \\
& \int x^{\alpha} g(x) d x=\int x^{\alpha} f_{j}(x) d x=0, \quad \text { if } \alpha \in \mathbb{N}^{3}, \quad 0 \leq|\alpha|<\delta-\frac{3}{2},
\end{aligned}
$$

and where the series converges a.e. in $\mathbb{R}^{3}$ and in $L_{\delta}^{2}$.

Proof. The result is obvious for $0 \leq \delta \leq \frac{3}{2}$, so we may assume $\delta>\frac{3}{2}$. We start with a bad choice, namely

$$
\tilde{g}(x)=f(x) I_{|x| \leq 1} \quad \text { and } \quad \tilde{f}_{j}(x)=f(x) I_{2^{j} \leq|x| \leq 2^{j+1}} \quad(j=0,1, \ldots),
$$

where $I$ denotes the indicator function. Letting $\tilde{f}_{-1}=\tilde{g}$, we set

$$
J(j, \alpha)=\int x^{\alpha} \tilde{f}_{j}(x) d x .
$$

Since $|\alpha|<\delta-\frac{3}{2}$, the series $\sum_{j} J(j, \alpha)$ converges and $\sum_{j=-1}^{\infty} J(j, \alpha)=0$. We now introduce a family of functions $\psi_{\beta} \in C_{0}^{\infty}\left(\mathbb{R}^{3}\right)$, supported in $\frac{1}{2} \leq|x| \leq 1$ and such that

$$
\int x^{\alpha} \psi_{\beta}(x) d x=\delta_{\alpha, \beta} \quad\left(\alpha, \beta \in \mathbb{N}^{3}, \quad|\alpha|,|\beta|<\delta-\frac{3}{2}\right),
$$

with $\delta_{\alpha, \beta}=0$ or 1 if $\alpha \neq \beta$ or $\alpha=\beta$, respectively (we may define $\psi_{\beta}$, e.g., through the tensorial product of suitable $C_{0}^{\infty}(\mathbb{R})$ functions).

Now let

$$
c(j, \alpha) \equiv J(j, \alpha)+J(j+1, \alpha)+\cdots,
$$

and set, for $j=-1,0, \ldots$,

$f_{j}(x)=\tilde{f}_{j}(x)-\sum_{\beta}\left(c(j, \beta) 2^{-(3+|\beta|) j} \psi_{\beta}\left(2^{-j} x\right)-c(j+1, \beta) 2^{-(3+|\beta|)(j+1)} \psi_{\beta}\left(2^{-j-1} x\right)\right)$,

the summation being taken over all $\beta \in \mathbb{N}^{3}$ such that $0 \leq|\beta|<\delta-\frac{3}{2}$.

Since $|J(j, \alpha)| \leq 2^{j|\alpha|} 2^{3 j / 2-j \delta} \tilde{\epsilon}_{j}$ for some $\tilde{\epsilon}_{j} \in \ell^{2}(\mathbb{N})$, we have $|c(j, \alpha)| \leq$ $2^{j|\alpha|} 2^{3 j / 2-j \delta} \bar{\epsilon}_{j}$, with $\bar{\epsilon}_{j} \in \ell^{2}(\mathbb{N})$. One now easily checks that $\sum_{j=-1}^{\infty} f_{j}=\sum_{j=-1}^{\infty} \tilde{f}_{j}=$ $f$ a.e. in $\mathbb{R}^{3}$ and that $g$ and $f_{j}$ satisfy $(2.1)$.

The converse is immediate. Note that if $g$ and $f_{j}$ satisfy the above conditions, then $\sum f_{j}$ must converge also in the $L^{1}\left(\mathbb{R}^{3}, w_{\delta}(x) d x\right)$ norm $\left(\delta>\frac{3}{2}\right)$ by Hölder's inequality, and this ensures the condition on the moments of $f$. Lemma 2.1 follows.

A similar decomposition applies to $Z_{\delta}$. 
Lemma 2.2. Let $\delta \geq 0$. We have $f \in Z_{\delta}$ if and only if

$$
f=g+\sum_{j=0}^{\infty} f_{j}
$$

with

$$
\begin{array}{ll}
\operatorname{supp} g \subset\{|x| \leq 1\}, & \operatorname{supp} f_{j} \subset\left\{2^{j-2} \leq|x| \leq 2^{j+2}\right\}, \\
g, \nabla g, \Delta g \in L^{2}\left(\mathbb{R}^{3}\right), & \left\|f_{j}\right\|_{2} \leq \epsilon_{j} 2^{2 j} 2^{-j \delta}, \quad \epsilon_{j} \in \ell^{2}(\mathbb{N}), \\
\left\|\nabla f_{j}\right\|_{2} \leq \bar{\epsilon}_{j} 2^{j} 2^{-j \delta}, & \left\|\Delta f_{j}\right\|_{2} \leq \tilde{\epsilon}_{j} 2^{-j \delta}, \quad \bar{\epsilon}_{j}, \tilde{\epsilon}_{j} \in \ell^{2}(\mathbb{N}),
\end{array}
$$

and where the series converges a.e. in $\mathbb{R}^{3}$ and in $Z_{\delta}$.

If $\delta>\frac{7}{2}$, then $Z_{\delta}$ is continuously embedded in $L^{1}\left(\mathbb{R}^{3}\right)$. In this case we have $\int f=0$ if and only if we may choose $g$ and $f_{j}$ satisfying, in addition, $\int g=\int f_{j}=0$ $(j=0,1, \ldots)$.

Proof. It is obvious that if $(2.2)$ holds, then $f=g+\sum_{j=0}^{\infty} f_{j}$ belongs to $Z_{\delta}$. Conversely, let $\varphi$ and $\psi$ be two compactly supported smooth functions, such that 0 does not belong to the support of $\psi$ and $1 \equiv \varphi(x)+\sum_{j=0}^{\infty} \psi\left(2^{-j} x\right)$. If we set $g(x)=f(x) \varphi(x)$ and $f_{j}(x)=f(x) \psi\left(2^{-j} x\right)$, then we have $\nabla f_{j}(x)=\psi\left(2^{-j} x\right) \nabla f(x)+$ $2^{-j}(\nabla \psi)\left(2^{-j} x\right) f(x)$ and

$$
\Delta f_{j}(x)=\psi\left(2^{-j} x\right) \Delta f(x)+2^{-j+1}(\nabla \psi)\left(2^{-j} x\right) \cdot \nabla f(x)+2^{-2 j}(\Delta \psi)\left(2^{-j} x\right) f(x) .
$$

Decomposition (2.2) then directly follows from the definition of $Z_{\delta}$.

If $\delta>\frac{7}{2}$, then $Z_{\delta} \subset L^{1}\left(\mathbb{R}^{3}\right)$ as checked with Hölder's inequality. In this case, when $\int f=0$, we can modify the definition of $g$ and $f_{j}$ reproducing the proof of Lemma 2.1 (with $|\alpha|=|\beta|=0$ ) and get the vanishing integral conditions. Lemma 2.2 follows.

We finish our study of $Z_{\delta}$ with the following lemma.

Lemma 2.3. Let $\delta \geq \frac{1}{2}$. Then $Z_{\delta}$ is an algebra with respect to the pointwise product. More precisely, if $f$ and $h$ belong to $Z_{\delta}$, then $f h \in Z_{2 \delta-1 / 2} \subset Z_{\delta}$.

Proof. The condition $\delta \geq \frac{1}{2}$ ensures that if $f \in Z_{\delta}$, then $f$ vanishes at infinity. Indeed, we have the following bound:

$$
|f(x)| \leq C(1+|x|)^{-\delta+1 / 2} \epsilon(x),
$$

where $\epsilon(x)$ is a bounded function vanishing at infinity. This is seen by applying Lemma 2.2 and writing, for $x \in \operatorname{supp} f_{j}, f_{j}(x)=\int|x-y|^{-1} \Delta f_{j}(y) d y$. Then applying Hölder's inequality and the last of $(2.2)$ we get for $x \in \operatorname{supp} f_{j},\left|f_{j}(x)\right| \leq \epsilon_{j} 2^{-j \delta} 2^{j / 2}$ with $\epsilon_{j} \in \ell^{2}(\mathbb{N})$ and our claim follows.

Another useful estimate (which follows interpolating $\nabla f_{j}$ between $\left\|\Delta f_{j}\right\|_{2}$ and $\left.\left\|f_{j}\right\|_{\infty}\right)$ is

$$
\left(\int_{2^{j} \leq|x| \leq 2^{j+1}}|\nabla f(x)|^{4} d x\right)^{1 / 4} \leq \epsilon_{j} 2^{j / 4-j \delta}, \quad \text { with } \epsilon_{j} \in \ell^{2}(\mathbb{N}) .
$$

Using this, we immediately see that if $f$ and $h$ belong to $Z_{\delta}$, then $f h \in$ $L^{2}\left(\mathbb{R}^{3},\left(1+|x|^{2}\right)^{2 \delta-5 / 2} d x\right), \nabla(f h) \in L^{2}\left(\mathbb{R}^{3},\left(1+|x|^{2}\right)^{2 \delta-3 / 2} d x\right)$, and $\Delta(f h) \in$ $L^{2}\left(\mathbb{R}^{3},\left(1+|x|^{2}\right)^{2 \delta-\frac{1}{2}} d x\right)$. Therefore $f h \in Z_{2 \delta-1 / 2} \subset Z_{\delta}$ and, moreover,

$$
\|f h\|_{Z_{2 \delta-1 / 2}} \leq C\|f\|_{Z_{\delta}}\|h\|_{Z_{\delta}} .
$$


3. Characterization of molecule spaces. We defined the molecule space $X_{\delta}$ in the introduction for $\delta>\frac{3}{2}$. These spaces can be defined also for $\frac{1}{2}<\delta \leq \frac{3}{2}$ by simply dropping the moment conditions on $\Delta f$ (it should be observed that the embedding $X_{\delta} \subset \Delta^{-1} \mathcal{H}^{1}\left(\mathbb{R}^{3}\right)$ breaks down for $\delta \leq \frac{3}{2}$, but for the sake of brevity we refer to $X_{\delta}$ as a "molecule space" also in this case). In this section we study the relation between the spaces $Z_{\delta}$ and $X_{\delta}$.

Proposition 3.1. We have

$$
\begin{array}{ll}
X_{\delta}=Z_{\delta}, \quad \text { if } \frac{1}{2}<\delta<\frac{7}{2} \quad \text { and } \quad \delta \neq \frac{3}{2}, \frac{5}{2}, \\
X_{\delta}=Z_{\delta} \cap\left\{f \in L^{1}\left(\mathbb{R}^{3}\right): \int f=0\right\}, \quad \text { if } \frac{7}{2}<\delta<\frac{9}{2}
\end{array}
$$

(with norm equivalence).

Note that $\delta=\frac{3}{2}, \frac{5}{2}, \frac{7}{2}$ are excluded. The proof of Proposition 3.1 will only give $Z_{\delta} \subset X_{\delta}$ in this case. Let us shed some light on this point with an example. Let $j, k=1,2,3$ and $f \in L^{2}\left(\mathbb{R}^{3}\right)$ such that $\Delta f=\partial_{j} \partial_{k} g$, where $g(x)=(4 \pi)^{-3 / 2} e^{-|x|^{2} / 4}$. Then $f \in X_{7 / 2}$ but $f \notin X_{\delta}$ for $\delta>\frac{7}{2}$, since $\int x_{j} x_{k} \Delta f(x) d x \neq 0$. Moreover, computing the inverse Fourier transform as in [17] from the identity $\widehat{f}(\xi)=\xi_{j} \xi_{k}|\xi|^{-2} e^{-|\xi|^{2}}$, one checks after some computations that the integral $\int|f(x)|^{2}\left(1+|x|^{2}\right)^{3 / 2} d x$ diverges so that $f \notin Z_{7 / 2}$.

Proof of Proposition 3.1. Throughout the proof $\alpha \in \mathbb{N}^{3}$. Note that because of our restrictions $\delta-\frac{3}{2}$ is not an integer.

First step: The embedding $X_{\delta} \subset Z_{\delta}$. Let $f \in X_{\delta}$. Applying Lemma 2.1 to $\Delta f$ and using the fact that $f$ vanishes at infinity, we see that we may write

$$
f=\frac{c}{|x|} * p+\sum_{j=0}^{\infty} \frac{c}{|x|} * q_{j}
$$

$c$ being an absolute constant. Here $p$ and $q_{j}$ are compactly supported $L^{2}$-functions, satisfying

$$
\begin{aligned}
& \operatorname{supp} p \subset\{|x| \leq 1\}, \\
& \operatorname{supp} q_{j} \subset\left\{2^{j-1} \leq|x| \leq 2^{j+1}\right\}, \\
& \left\|q_{j}\right\|_{2} \leq \epsilon_{j} 2^{-j \delta}, \quad \epsilon_{j} \in \ell^{2}(\mathbb{N}), \\
& \int x^{\alpha} p(x) d x=\int x^{\alpha} q_{j}(x) d x=0, \quad \text { if }|\alpha|<\delta-\frac{3}{2} .
\end{aligned}
$$

Let us show that, for all $f \in X_{\delta}$ and $2^{j} \leq|x| \leq 2^{j+1}$, we have

$$
|f(x)| \leq \bar{\epsilon}_{j} 2^{j / 2} 2^{-j \delta}, \quad \text { with } \bar{\epsilon}_{j} \in \ell^{2}(\mathbb{N}) .
$$

To prove (3.5) we set $P=\frac{1}{|\cdot|} * p, Q_{j}=\frac{1}{|\cdot|} * q_{j}(j=0,1, \ldots)$, and $d=\left[\delta-\frac{3}{2}\right]$ (we set $d=-1$ if $\frac{1}{2}<\delta<\frac{3}{2}$ ). Then we have

$$
\begin{array}{lrl}
\left|Q_{j}(x)\right| \leq C \epsilon_{j} 2^{-j(\delta-1 / 2)}, & \text { if }|x| \leq 4 \cdot 2^{j}, \\
\left|Q_{j}(x)\right| \leq C|x|^{-(d+2)} \epsilon_{j} 2^{(d+5 / 2-\delta) j}, & \text { if }|x| \geq 4 \cdot 2^{j} .
\end{array}
$$

The first bound follows from the localization of $q_{j}$ and Hölder's inequality. Let us prove (3.7); we start by introducing the Taylor polynomial $y \mapsto T_{x}(y)$ of degree $d$ 
centered at $x$ of the function $1 /|y|$ (we set $T_{x}(y) \equiv 0$ for $\frac{1}{2}<\delta<\frac{3}{2}$ ). Then for $|x| \geq 4 \cdot 2^{j}$ and $y \in \operatorname{supp} q_{j}$ we may write, using the last of (3.4),

$$
\left|Q_{j}(x)\right|=\left|\int\left(\frac{1}{|x-y|}-T_{x}(-y)\right) q_{j}(y) d y\right| \leq C|x|^{-d-2} \int|y|^{d+1}\left|q_{j}(y)\right| d y .
$$

Here the inequality follows from the Taylor formula and the fact that the $(d+1)$-order derivatives of $y \mapsto 1 /|y|$ are bounded in a ball centered at $x$ and radium $|x| / 2$, up to a constant, by $|x|^{-d-2}$. The bound (3.7) now follows from Hölder's inequality.

Similar arguments allow us to see that $|P(x)| \leq C(1+|x|)^{-(d+2)}$. Summing up on these inequalities immediately yields (3.5).

Condition (3.6) also ensures that $\int_{|x| \leq 1}|f|^{2}$ is finite. Then using (3.5) we get

$$
\int|f(x)|^{2}\left(1+|x|^{2}\right)^{\delta-2} d x<\infty .
$$

We now need some bounds for $\nabla f$. We start from $-\nabla f(x)=\frac{c x}{|x|^{3}} * p+\sum_{j=0}^{\infty} \frac{c x}{|x|^{3}} *$ $q_{j}$, and we set $R_{j}=\left(x /|x|^{3}\right) * q_{j}(j=0,1, \ldots)$ and $R_{-1}=\left(x /|x|^{3}\right) * p$. Then for $j \geq-1$,

$$
\begin{aligned}
& \left|R_{j}(x)\right| \leq C|x|^{-(d+3)} \epsilon_{j} 2^{(d+5 / 2-\delta) j}, \quad \text { if }|x| \geq 4 \cdot 2^{j}, \\
& \left(\int_{|x| \leq 4 \cdot 2^{j}}\left|R_{j}(x)\right|^{4} d x\right)^{1 / 4} \leq C \epsilon_{j} 2^{j / 4} 2^{-j \delta}
\end{aligned}
$$

Indeed, (3.9) again easily follows using the vanishing of the moments of $q_{j}$ and the Taylor formula. The proof of (3.10) deserves a more detailed explanation: for $|x| \leq$ $4 \cdot 2^{j}$ we write

$$
\frac{x}{|x|^{3}} * q_{j}(x)=\theta_{j} * q_{j}(x), \quad \text { where } \quad \theta_{j}(x)=\frac{x}{|x|^{3}} I_{\left\{|x| \leq 6 \cdot 2^{j}\right\}} .
$$

Then (3.10) comes from $\left\|\theta_{j} * q_{j}\right\|_{4} \leq\left\|\theta_{j}\right\|_{4 / 3}\left\|q_{j}\right\|_{2} \leq C 2^{j / 4}\left\|q_{j}\right\|_{2}$.

Now, for $j \geq 1$ we write $-\frac{1}{c} \nabla f=\sum_{k=-1}^{j-2} R_{k}+\sum_{k=j-1}^{\infty} R_{k} \equiv A_{j}+B_{j}$. Using (3.9) we get

$$
\left(\int_{2^{j} \leq|x| \leq 2^{j+1}}\left|A_{j}(x)\right|^{2} d x\right)^{1 / 2} \leq C \tilde{\epsilon}_{j} 2^{j} 2^{-j \delta}, \quad \text { with } \tilde{\epsilon}_{j} \in \ell^{2}(\mathbb{N}) .
$$

On the other hand, applying Hölder and Minkowski inequalities and (3.10) yields

$$
\left(\int_{2^{j} \leq|x| \leq 2^{j+1}}\left|B_{j}(x)\right|^{2} d x\right)^{1 / 2} \leq C^{\prime} \tilde{\epsilon}_{j} 2^{j} 2^{-j \delta}, \quad \text { with } \tilde{\epsilon}_{j} \in \ell^{2}(\mathbb{N}) .
$$

Since we obviously have $\int_{|x| \leq 2}|\nabla f|^{2}<\infty$, we thus see that

$$
\int|\nabla f(x)|^{2}\left(1+|x|^{2}\right)^{\delta-1} d x<\infty
$$

This last inequality, condition (3.8), and the definition of the $X_{\delta}$ norm yield the injection $X_{\delta} \subset Z_{\delta}$.

Second step: The elements of $X_{\delta}, \frac{7}{2}<\delta<\frac{9}{2}$ have vanishing integral. Assume now $\frac{7}{2}<\delta<\frac{9}{2}$. Then the moments of $p$ and $q_{j}$ vanish up to the order two. Moreover, 
our previous estimates imply that $P$ and $Q_{j}$ belong to $L^{1}\left(\mathbb{R}^{3}\right)$. We thus see, e.g., via the Fourier transform (using the fact that $\widehat{p}(\xi)$ and $\widehat{q}_{j}(\xi)$ vanish at the origin together with their derivatives up to the order two and letting $\xi \rightarrow 0$ ) that $\int P(x) d x=$ $\int Q_{j}(x) d x=0$ for all positive integers $j$. Moreover, the series $\sum Q_{j}$ converges in the $L^{1}$-norm by (3.6)-(3.7) yielding $\int f=0$.

Third step: The converse inclusion. Let $f \in Z_{\delta}$. In the case $\frac{7}{2}<\delta<\frac{9}{2}$ we assume $\int f=0$. The bound $\|f\|_{X_{\delta}}<\infty$ is obvious. By Lemma 2.2 we have $f=g+\sum_{j=0}^{\infty} f_{j}$, such that (2.2) holds (with $\int g=\int f_{j}=0$ for all $j \geq 0$ if $\frac{7}{2}<\delta<\frac{9}{2}$ ).

We claim that

$$
\int x^{\alpha} \Delta g(x) d x=\int x^{\alpha} \Delta f_{j}(x) d x=0, \quad 0 \leq|\alpha|<\delta-\frac{3}{2},
$$

for all $j \geq 0$ (there are no moment conditions for $\frac{1}{2}<\delta<\frac{3}{2}$ ). Indeed, since $g$ and $f_{j}$ are compactly supported, when applying the Green formula all the boundary terms vanish and we obtain (e.g., for $f_{j}$, when $\frac{7}{2}<\delta<\frac{9}{2}$ )

$$
\int x^{\alpha} \Delta f_{j}(x) d x=2 \int f_{j}(x) d x, \quad \text { if } \quad x^{\alpha}=x_{1}^{2}, x_{2}^{2}, \text { or } x_{3}^{2},
$$

and $\int x^{\alpha} \Delta f_{j}(x) d x=0$; otherwise $\left(|\alpha| \leq\left[\delta-\frac{3}{2}\right]\right)$. Our claim then follows.

Moreover, by Hölder's inequality,

$$
\sum_{j=0}^{\infty}\left\|x^{\alpha} \Delta f_{j}\right\|_{1}<\infty, \quad 0 \leq|\alpha|<\delta-\frac{3}{2} .
$$

Summing on $j$ we get $\int x^{\alpha} \Delta f(x) d x=0$.

To conclude that $f \in X_{\delta}$ it remains to check that $f$ vanishes at infinity. This was done in (2.3).

4. Proof of Theorem 1.1. The boundedness of the operator $\nabla e^{t \Delta} \mathbb{P}$ in $X_{\delta}$ $\left(\frac{3}{2}<\delta<\frac{9}{2}\right), \delta \neq \frac{5}{2}, \frac{7}{2}$ is a fundamental step of [12]. Lemma 4.1 provides a short proof of this fact. Our main tool will be the realization of homogeneous Sobolev spaces introduced by Bourdaud. Note that $\nabla e^{t \Delta} \mathbb{P}$ is a matrix operator acting on vector fields. But its matricial structure has no special role in what follows, since we shall establish all the relevant estimates componentwise.

Lemma 4.1. Let $\frac{1}{2}<\delta<\frac{9}{2}, \delta \neq \frac{3}{2}, \frac{5}{2}, \frac{7}{2}$. The operator $\nabla e^{t \Delta} \mathbb{P}$ is bounded from $Z_{\delta}$ to $X_{\delta}$ for all $t>0$, with operator norm $O\left(t^{-1 / 2}\right)$ as $t \rightarrow 0$.

Proof. Let $f \in Z_{\delta}$. If $\frac{7}{2}<\delta<\frac{9}{2}$, then we introduce a function $h$ such that

$$
f(x)=c g(x)+h(x), \quad \text { where } g(x)=(4 \pi)^{-3 / 2} e^{-|x|^{2} / 4}
$$

and the constant $c$ is chosen in a such way that $\int h(x) d x=0$. If, instead, $\frac{1}{2}<\delta<\frac{7}{2}$, $\delta \neq \frac{3}{2}, \frac{5}{2}$, then we simply set $f(x)=h(x)$. In any case, we deduce from Proposition 3.1 that $h \in X_{\delta}$ and $\|h\|_{X_{\delta}} \leq C\|f\|_{Z_{\delta}}$ for some constant $C$ depending only on $\delta$.

We start showing that $\nabla e^{t \Delta} \mathbb{P} g$ belongs to $X_{\delta}$ for all $0 \leq \delta<\frac{9}{2}$. Note that the components of $\left.\left(\nabla e^{t \Delta} \mathbb{P} g\right) \hat{(} \xi\right)$ are given by

$$
\mathrm{i} \xi_{h}\left(\delta_{j, k}-\frac{\xi_{j} \xi_{k}}{|\xi|^{2}}\right) \exp \left(-(t+1)|\xi|^{2}\right) \quad\left(j, h, k=1,2,3, \quad \delta_{j, k}=0 \text { or } 1\right),
$$


and the inverse Fourier transform can be easily computed (see, e.g., [17]). We immediately find that $\nabla e^{t \Delta} \mathbb{P} g$ is a smooth function in $\mathbb{R}^{3}$, such that

$$
\left|\partial^{\alpha} \nabla e^{t \Delta} \operatorname{P} g(x)\right| \leq C_{\alpha}(1+|x|)^{-(4+|\alpha|)} \quad \text { for all } \alpha \in \mathbb{N}^{3} .
$$

This bound implies that $e^{t \Delta} \mathbb{P} \nabla g \in Z_{\delta}$, for $0 \leq \delta<\frac{9}{2}$. But $\int \nabla e^{t \Delta} \mathbb{P} g=0$ (the Fourier transform of the integrand vanishes at the origin) and thus $\nabla e^{t \Delta} \mathbb{P} g$ belongs, more precisely, to $X_{\delta}$.

Let us now prove that $\nabla e^{t \Delta} \mathbb{P} h$ does also belong to $X_{\delta}$. The only difficulty is for $\frac{3}{2}<\delta<\frac{9}{2}, \delta \neq \frac{5}{2}, \frac{7}{2}$. Indeed, if $\frac{1}{2}<\delta<\frac{3}{2}$, then one observes that the weight $\left(1+|x|^{2}\right)^{\delta}$ belongs to the Muckenhoupt class $A_{2}$; see [18]. This implies that $\mathbb{P}$, and more generally the Riesz transforms, are bounded in $L^{2}\left(\mathbb{R}^{3},\left(1+|x|^{2}\right)^{\delta} d x\right)$. Since $\nabla e^{t \Delta} \mathbb{P}$ and the Laplacian commute, the result easily follows applying this remark to $\Delta h$.

To deal with the case $\delta>\frac{3}{2}$, we start by recalling that the Sobolev space $H^{\delta}$ is defined by

$$
\|q\|_{H^{\delta}}^{2} \equiv \int|\widehat{q}(\xi)|^{2}\left(1+|\xi|^{2}\right)^{\delta} d \xi
$$

and that $H^{\delta} \subset C^{\delta-3 / 2}$ (the Hölder-Zygmund space). Thus, stating that $h$ belongs to $X_{\delta}$ is equivalent to stating that

$$
q(\xi) \equiv|\xi|^{2} \widehat{h}(\xi) \in H^{\delta} \quad \text { and } \quad \partial^{\alpha} q(0)=0 \quad \text { for all } 0 \leq|\alpha| \leq\left[\delta-\frac{3}{2}\right] .
$$

These two conditions on $q$ can be expressed by saying that $q$ belongs to $L^{2}\left(\mathbb{R}^{3}\right) \cap \dot{H}_{\text {rel }}^{\delta}$, where $\dot{H}_{r e l}^{\delta}$ is the realization of the homogeneous Sobolev space $\dot{H}^{\delta}$ (see Bourdaud [3]). Recall that $\dot{H}_{\text {rel }}^{\delta}$ can be injected into $\mathcal{S}^{\prime}\left(\mathbb{R}^{3}\right.$ ) (this would not be true for $\dot{H}^{\delta}$, which is instead a space of tempered distributions modulo polynomials) and hence the notion of pointwise multipliers makes sense in the realized space. It follows from the result of [3] that $m(\xi) \equiv \xi_{j} /|\xi|$ is a multiplier for $\dot{H}_{\text {rel }}^{\delta}$ (any homogeneous function of degree 0 which is smooth outside the origin is indeed a multiplier for this space).

Since $h \in X_{\delta}$, the components of $|\xi|^{2} \widehat{\mathbb{P h}}(\xi)$, which are given by $\left(\delta_{j, k}-\xi_{j} \xi_{k}|\xi|^{-2}\right) q(\xi)$, belong to $L^{2}\left(\mathbb{R}^{3}\right) \cap \dot{H}_{\text {rel }}^{\delta}$. Hence, $\mathbb{P} h \in X_{\delta}$. Moreover, $\mathrm{i} \xi_{h} e^{-t|\xi|^{2}} \in \mathcal{S}\left(\mathbb{R}^{3}\right)$ is also a multiplier of $\dot{H}_{\text {rel }}^{\delta}$ (with norm $c / \sqrt{t}$ ). Then we get $\left\|\nabla e^{t \Delta} \mathbb{P} f\right\|_{X_{\delta}} \leq C t^{-1 / 2}\|f\|_{Z_{\delta}}$ and Lemma 4.1 is thus proven.

Our last lemma deals with the case $\delta=\frac{1}{2}$.

LEMma 4.2. The operator $\nabla e^{t \Delta} \mathbb{P}$ is bounded in $Z_{1 / 2}$ for all $t>0$, with operator norm $O\left(t^{-1 / 2}\right)$ as $t \rightarrow 0$.

Proof. Following Miyakawa's notations, we denote by $F(x, t)$ the kernel of the operator $\nabla e^{t \Delta} \mathbb{P}$. Then we know that $F(x, t)=t^{-2} \Phi(x / \sqrt{t})$, where $\Phi$ is smooth in $\mathbb{R}^{3}$ and $|\Phi(x)| \leq C(1+|x|)^{-4}$ (see again [17] for more details). Let us show that $\|\Phi * f\|_{Z_{1 / 2}} \leq C\|f\|_{Z_{1 / 2}}$. Then the conclusion will follow from a simple rescaling argument.

Let $f \in Z_{1 / 2}$ and write $f=g+\sum_{j=0}^{\infty} f_{j}$, where $g$ and $f_{j}$ satisfy (2.2). We also know that $\left|f_{j}(x)\right| \leq \epsilon_{j}$ for all $x \in \operatorname{supp} f_{j}$ (we saw this right after (2.3)), with $\epsilon_{j} \in$ $\ell^{2}(\mathbb{N})$. We obviously have $\Phi * g \in Z_{1 / 2}$ and $\Phi * f \in L^{\infty}\left(\mathbb{R}^{3}\right)$. Now let $2^{k} \leq|x| \leq 2^{k+1}$ $(k \in \mathbb{N}, k \geq 3)$. Using the decay of $\Phi$ we see that $\Phi * f_{j}$ is bounded by $C \epsilon_{j} 2^{-4 k} 2^{3 j}$ $(j \leq k-3)$ and $C \epsilon_{j} 2^{-j}(j \geq k+3)$. Thus $|\Phi * f(x)| \leq \tilde{\epsilon}_{k}$, with $\tilde{\epsilon}_{k} \in \ell^{2}(\mathbb{N})$, and we conclude that $\int|\Phi * f(x)|^{2}\left(1+|x|^{2}\right)^{-3 / 2} d x$ is finite. 
Moreover, by Young's inequality,

$$
\begin{aligned}
\int \mid \nabla & \left.* f(x)\right|^{2}\left(1+|x|^{2}\right)^{-1 / 2} d x \\
& \leq C\left(\int|\Phi(x)|(1+|x|)^{1 / 2} d x\right)^{2} \int|\nabla f(x)|^{2}\left(1+|x|^{2}\right)^{-1 / 2} d x, \\
\int|\Delta \Phi * f(x)|^{2}\left(1+|x|^{2}\right)^{1 / 2} d x & \leq C\left(\int|\Phi(x)|(1+|x|)^{1 / 2} d x\right)^{2} \int|\Delta f(x)|^{2}\left(1+|x|^{2}\right)^{1 / 2} d x
\end{aligned}
$$

(we also used $(1+|x|)^{-1 / 2} \leq c(1+|y|)^{-1 / 2}(1+|x-y|)^{1 / 2}$ in the first inequality and $(1+|x|)^{1 / 2} \leq c(1+|y|)^{1 / 2}(1+|x-y|)^{1 / 2}$ in the second inequality). Lemma 4.2 now follows.

Proof of Theorem 1.1. The proof is based on the application of Kato's standard iteration argument in the space $C\left([0, T], Z_{\delta}\right)$. Let us write (IE) in the compact form $u(t)=e^{t \Delta} a-B(u, u)$, where $B(u, v)=\int_{0}^{t} \nabla \cdot e^{(t-s) \Delta} \mathbb{P}(u \otimes v)(s) d s$.

By Lemmas 4.1 and 4.2 , and using the fact that $Z_{\delta}$ is a pointwise algebra for $\delta \geq \frac{1}{2}$, we see that the bilinear operator $B$ is bounded in $C\left([0, T], Z_{\delta}\right)$ for $\frac{1}{2} \leq \delta<\frac{9}{2}$, $\delta \neq \frac{3}{2}, \frac{5}{2}, \frac{7}{2}$, (the continuity with respect to the time variable is straightforward). Moreover, $\|B(u, v)\|_{\delta} \leq C_{T}\|u\|_{\delta}\|v\|_{\delta}$, where $\|w\|_{\delta} \equiv \sup _{t \in[0, T]}\|w(t)\|_{Z_{\delta}}$ and $C_{T}=$ $O\left(T^{1 / 2}\right)$ as $t \rightarrow 0$. We get the same conclusion for $\delta=\frac{3}{2}, \frac{5}{2}, \frac{7}{2}$ if we use the continuous embedding $Z_{\delta} \subset Z_{\delta^{\prime}}\left(\delta \geq \delta^{\prime}\right)$ and the stronger version of Lemma 2.3. For example,

$$
\|B(u, v)\|_{3 / 2} \leq C\|B(u, v)\|_{2} \leq C T^{1 / 2}\|u\|_{5 / 4}\|v\|_{5 / 4} \leq C T^{1 / 2}\|u\|_{3 / 2}\|v\|_{3 / 2},
$$

and a similar argument can be used for $\delta=\frac{5}{2}, \frac{7}{2}$.

Since $e^{t \Delta} a$ belongs to $C\left([0, T], Z_{\delta}\right)$ if $a \in Z_{\delta}$ for all $\delta \geq 0$, as is easily checked, we see that the fixed point argument applies in $C\left([0, T], Z_{\delta}\right)$, at least if $T>0$ is small enough.

We state as a corollary a slight improvement of Furioli and Terraneo's theorem.

Corollary 4.3. Let $\frac{1}{2}<\delta<\frac{9}{2}$ and $a \in X_{\delta}$. Then there exists $T>0$ such that (IE) can be uniquely solved in $C\left([0, T], X_{\delta}\right)$.

Proof. The result is true for $\frac{1}{2}<\delta<\frac{7}{2}$ and $\delta \neq \frac{3}{2}, \frac{5}{2}$ because of the identification between $X_{\delta}$ and $Z_{\delta}$. For $\frac{7}{2}<\delta<\frac{9}{2}$ we use the fact that every divergence-free vector field which is in $L^{1}\left(\mathbb{R}^{3}\right)$ must have a vanishing integral. Hence the existence and unicity result for (IE) in $X_{\delta}$ again follows from the corresponding result in $Z_{\delta}$ and the last conclusion of Proposition 3.1.

In the case $\delta=\frac{3}{2}$ we can observe that if $u, v \in C\left([0, T], X_{3 / 2}\right)$, then $B(u, v)$ belongs, e.g., to $C\left([0, T], Z_{2}\right)$, which is contained in $C\left([0, T], X_{3 / 2}\right)$. A similar argument can be used for $\delta=\frac{5}{2}, \frac{7}{2}$ and the conclusion easily follows.

As claimed in the introduction, the restriction $\delta<\frac{9}{2}$ cannot be removed in Theorem 1.1. Indeed, if $u$ is a solution to (IE) such that $u \in C\left([0, T], Z_{9 / 2}\right)$, for some $T>0$, then the initial datum must satisfy the conditions of Dobrokhotov and Shafarevich: $\int\left(a_{j} a_{k}\right)=0$ if $j \neq k$ and $\int a_{1}^{2}=\int a_{2}^{2}=\int a_{3}^{2}$. This is due to the fact that the localization condition $a \in L^{2}\left(\mathbb{R}^{3},\left(1+|x|^{2}\right)^{5 / 2} d x\right)$ is not conserved during the evolution (see [6]). For the same reason, the condition $a \in X_{\delta}, \delta>\frac{9}{2}$ breaks down (in general). But we do not know if the condition $a \in X_{9 / 2}$ (or $a \in X_{1 / 2}$ ) propagates.

We conclude by observing that spatially localized flows $u(t)$, belonging to $Z_{\delta}$ and $X_{\delta}$ with $\delta>\frac{9}{2}$, do, however, exist. Examples of such flows can be found in [4]. 
Acknowledgment. The author would like to thank the three referees for their remarks and useful comments, which helped him to improve the first version of this paper in a number of ways.

\section{REFERENCES}

[1] C. Amrouche, V. Girault, M. E. Schonbek, and T. P. Schonbek, Pointwise decay of solutions and of higher derivatives to Navier-Stokes equations, SIAM J. Math. Anal., 31 (2000), pp. 740-753.

[2] H.-O. BaE AND B. J. Jin, Temporal and Spatial Decays for the Navier-Stokes Equation, preprint, Ajou University, Suwon, Republic of Korea.

[3] G. Bourdaud, Localisation et multiplicateurs des espaces de Sobolev homogènes, Manuscripta Math., 60 (1988), pp. 93-130.

[4] L. Brandolese, Space-time decay of Navier-Stokes flows invariant under rotations, Math. Ann., 329 (2004), pp. 685-706.

[5] L. Brandolese, Weighted $L^{2}$-spaces and strong solutions of the Navier-Stokes equations in $\mathbb{R}^{3}$, in Trends in Partial Differential Equations of Mathematical Physics, Progr. Nonlinear Differential Equations Appl. 61, Birkhäuser, Basel, 2005, pp. 27-35.

[6] L. Brandolese AND Y. MeYer, On the instantaneous spreading for the Navier-Stokes system in the whole space, ESAIM Control Optim. Calc. Var., 8 (2002), pp. 273-285.

[7] R. Coifman, P.-L. Lions, Y. Meyer, and S. Semmes, Compensated compactness and Hardy spaces, J. Math. Pures Appl., 72 (1993), pp. 247-286.

[8] R. Coifman And G. Weiss, Extension of the Hardy spaces and their use in analysis, Bull. Amer. Math. Soc., 83 (1977), pp. 569-645.

[9] R. FARwig AND H. Sohr, Weighted energy inequalities for the Navier-Stokes equations in exterior domains, Appl. Anal., 58 (1995), pp. 157-173.

[10] R. FARwig AND H. Sohr, Global estimates in weighted spaces of weak solutions of the NavierStokes equations in exterior domains, Arch. Math. (Basel), 67 (1996), pp. 319-330.

[11] G. Furioli, P.-G. Lemarié-Rieusset, and E. Terraneo, Unicité dans $L^{3}\left(\mathbb{R}^{3}\right)$ et d'autres espaces fonctionnels limites, Rev. Mat. Iberoamericana, 16 (2000), pp. 269-315.

[12] G. Furioli and E. Terraneo, Molecules of the Hardy space and the Navier-Stokes equations, Funkcial. Ekvac., 45 (2002), pp. 141-160.

[13] C. He, Weighted estimates for nonstationary Navier-Stokes equations, J. Differential Equations, 148 (1998), pp. 422-444.

[14] C. He And Z. XIN, On the decay properties of solutions to the nonstationary Navier-Stokes equations in $\mathbb{R}^{3}$, Proc. Roy. Soc. Edinburgh Sect. A, 131 (2001), pp. 597-619.

[15] P. G. Lemarié-Rieusset, Recent Developments in the Navier-Stokes Problem, Chapman \& Hall, CRC Press, Boca Raton, FL, 2002.

[16] T. Miyakawa, Application of Hardy space techniques to the time-decay problem for incompressible Navier-Stokes flows in $\mathbb{R}^{n}$, Funkcial. Ekvac., 41 (1998), pp. 383-434.

[17] T. MiYaKaWA, On space time decay properties of nonstationary incompressible Navier-Stokes flows in $\mathbb{R}^{n}$, Funkcial. Ekvac., 32 (2000), pp. 541-557.

[18] E. M. Stein, Harmonic Analysis. Real-Variable Methods, Orthogonality and Oscillatory Integrals, Princeton University Press, Princeton, NJ, 1993.

[19] F. Vigneron, Spatial decay of the velocity field of an incompressible viscous fluid in $\mathbb{R}^{d}$, Nonlinear Anal., to appear.

[20] A. Youssfi, Localisation des espaces de Besov homogènes, Indiana Univ. Math. J., 37 (1988), pp. $565-587$. 